\title{
CONTRIBUIÇÃO DE CONSTITUINTES DE SOLO À CAPACIDADE DE TROCA DE CÁTIONS OBTIDA POR DIFERENTES MÉTODOS DE EXTRAÇÃO ${ }^{(1)}$
}

\author{
Edson Campanhola Bortoluzzi ${ }^{(2)}$, Danilo dos Santos Rheinheimer ${ }^{(3)}$, \\ Claudia Petry ${ }^{(2)}$ \& João Kaminski ${ }^{(3)}$
}

\begin{abstract}
RESUMO
A capacidade de troca de cátions (CTC) é uma propriedade físico-química intrínseca aos constituintes minerais e orgânicos do solo. Apesar do uso de diferentes extratores e procedimentos, a CTC é normalmente expressa considerando apenas o controle ou não do pH na solução extratora. O objetivo deste trabalho foi discutir o significado da contribuição da matéria orgânica do solo prepresentada pelo carbono orgânico total (COT) e da argila à capacidade de troca de cátions de um Argissolo quando diferentes métodos estão envolvidos na determinação desse parâmetro. Para isso, utilizaram-se 75 amostras de um Argissolo Vermelho-Amarelo distrófico abrúptico da área do Departamento de Solos da Universidade Federal de Santa Maria, representando, em triplicata, cinco profundidades e cinco sistemas de uso e manejo de solo. A CTC efetiva $\left(\mathrm{CTC}_{\mathrm{E}}\right)$ foi estimada pelo cloreto de hexamina cobalto $\left(\mathrm{CTC}_{\mathrm{E}}\right.$ Cohex) e pela soma de cátions $\mathrm{Al}^{3+}, \mathrm{Ca}^{2+}, \mathrm{Mg}^{2+}$ e $\mathrm{K}^{+}\left(\mathrm{CTC}_{\mathrm{E}} \mathrm{SB}+\mathrm{Al}_{\mathrm{KCl}}\right)$, os três primeiros extraídos por $\mathrm{KCl}$ e o último por Mehlich-1; a CTC em pH 7,0 $\left(\mathrm{CTC}_{7}\right)$ foi estimada por acetato de amônio ( $\mathrm{CTC}_{7}$ Metson) e pela soma de cátions $\mathrm{Ca}^{2+}, \mathrm{Mg}^{2+}$ e $\mathrm{K}^{+} \mathrm{e} \mathrm{H}+\mathrm{Al}$ estimado pelo índice $\mathrm{SMP}\left(\mathrm{CTC}_{7} \mathrm{SB}+\mathrm{H}+\mathrm{Al}_{\mathrm{SMP}}\right)$. Os valores de $\mathrm{CTC}$ obtidos pelos diferentes métodos se relacionam entre si, com coeficientes de correlação linear simples acima de 0,93 . Os valores de $\mathrm{CTC}_{7}$ Metson são subestimados quando comparados com o método $\mathrm{CTC}_{7}\left(\mathrm{SB}+\mathrm{H}+\mathrm{Al}_{\mathrm{SMP}}\right)$. Nesse sentido, as contribuições da argila e do COT à $\mathrm{CTC}_{7}$ foram, respectivamente, menores para a $\mathrm{CTC}_{7}$ Metson, 19 e $256 \mathrm{cmol}_{\mathrm{c}} \mathrm{kg}^{-1}$, que para a $\mathrm{CTC}_{7}\left(\mathrm{SB}+\mathrm{H}+\mathrm{Al}_{\mathrm{SMP}}\right), 23$ e $399 \mathrm{cmol}_{\mathrm{c}} \mathrm{kg}^{-1}$. A contribuição dos constituintes de solo depende, então, do cátion extrator e da capacidade de extração dos métodos empregados.
\end{abstract}

Termos de indexação: argilominerais, matéria orgânica, cloreto de hexamina cobalto, acetato de amônio, $\mathrm{pH}$.

\footnotetext{
(1) Recebido para publicação em maio de 2008 e aprovado em março de 2009.

(2) Professor, Pesquisador da Faculdade de Agronomia e Medicina Veterinária da Universidade de Passo Fundo - UPF. Caixa Postal 611, CEP 99001-970 Passo Fundo (RS). E-mail: edsonb@upf.br; petry@upf.br

(3) Professor do Departamento de Solos, Centro de Ciências Rurais, Universidade Federal de Santa Maria - UFSM. Camobi, CEP 97105-900 Santa Maria (RS).E-mails: danilor@smail.ufsm.br; jkaminski@pq.cnpq.br
} 


\title{
SUMMARY: CONTRIBUTION OF SOIL CONSTITUENTS TO THE CATION EXCHANGE CAPACITY AS DETERMINED BY DIFFERENT EXTRACTION METHODS
}

\begin{abstract}
The cation exchange capacity (CEC) is a physicochemical property dependent on mineral and organic soil constituents. Despite the use of different procedures and extractors the CEC is normally expressed considering only the $\mathrm{pH}$ control or not of the extracted solution. This study aims to discuss the significance of the contribution of organic carbon and clay to the CEC of an Acrisol, using different determination methods. For this purpose, 75 samples of an abruptic Red-Yellow Acrisol from the Universidade Federal de Santa Maria campus were used, representing five layers and five land use and management systems, in triplicate. The $C E C_{E}$ was estimated by cobalt hexamine trichloride $\left(C E C_{E}\right.$ Cohex) and by the sum of cations $\mathrm{Al}^{3+}$, $\mathrm{Ca}^{2+}, \mathrm{Mg}^{2+}$, and $\mathrm{K}^{+}\left(C E C_{E} S B+A l_{K C l}\right)$, the first three extracted by $\mathrm{KCl}$ and the last by Mehlich-1; the $\mathrm{CEC}_{7}$ was estimated by ammonium acetate $\left(C E C_{7}\right.$ Metson) and the sum of bases $\mathrm{Ca}^{2+}$, $M^{2+}$ and $K^{+}$and $H+A l$ was estimated by the $S M P$ index $\left(C E C_{7} S B+H+A l_{S M P}\right)$. The $C E C$ values obtained with the different methods are correlated, with coefficients of determination over 0.93. The $\mathrm{CEC}_{7}$ Metson values are subestimated when compared with those by $\mathrm{CEC}_{7}$ $\left(S B+H+A l_{S M P}\right)$. The contribution of clay and organic carbon to $C E C_{7}$ was, respectively, 23 and $399 \mathrm{cmol}_{c} \mathrm{~kg}^{-1}$ for $\mathrm{CEC}_{7}\left(\mathrm{SB}+\mathrm{H}+\mathrm{Al} \mathrm{S}_{\mathrm{SMP}}\right)$ and 19 and $256 \mathrm{cmol}_{c} \mathrm{~kg}^{-1}$ for $\mathrm{CEC}_{7}$ Metson. The contribution of the soil constituents depends on the extracting cation and the performance of the extraction methods employed.
\end{abstract}

Index terms: clay minerals, organic matter, cobalt hexamine trichloride, ammonium acetate, $p H$.

\section{INTRODUÇÃO}

A quantidade de íons adsorvidos, com diferentes graus de energia, aos grupos funcionais de superfície dos constituintes de solos pode ser estimada por diversos métodos analíticos. Normalmente, os métodos exploram o poder de extração pela variação das soluções químicas, das suas concentrações, do $\mathrm{pH}$ da solução, do tempo de contato e de procedimentos laboratoriais (Barrow, 1999).

Fracionamentos químicos, como o proposto por Tessier et al. (1979), permitem extrair espécies químicas com energia de ligação sorbato-sorvente diferentes, estabelecendo-se assim a relação entre o teor extraído e o compartimento ou grupo funcional associado. Anderson \& Sposito (1992) modelaram a relação em que as cargas intrínsecas dos constituintes estão em equilíbrio químico com a magnitude dos íons trocáveis na solução do solo. Esse princípio é aplicado ao fracionamento de fósforo (Gatiboni et al., 2007) e K (Kaminski et al., 2007). No mesmo sentido, a eliminação seletiva de constituintes do solo, apesar de trabalhosa, também é utilizada com sucesso (Souza et al., 2007). Modelos estatísticos constituem-se em meio rápido e confiável na estimação da contribuição relativa dos constituintes de solo à capacidade de troca de cátions (CTC) (Helling et al., 1964; Bennema, 1966; Villiers \& Jackson, 1967) como também dos constituintes às cargas elétricas variáveis ou permanentes (Katou, 2002, Bortoluzzi et al., 2006).

A CTC como propriedade diagnóstica da retenção e troca de cátions do solo pode ser feita utilizando-se inúmeros métodos (Metson, 1956; Orsini \& Remy,
1976; Tedesco et al., 1995; Afnor, 1996; Ciesielski \& Sterckeman, 1997). Extratores não tamponados devem permitir estimar a CTC do solo em seu estado natural de $\mathrm{pH}$ ao passo que extratores tamponados estimam a CTC em determinado $\mathrm{pH}$, normalmente mais elevado que o $\mathrm{pH}$ natural do solo. O uso de soluções extratoras compostas por um único cátion apresenta-se vantajoso na estimação da CTC em relação aos métodos que usam a soma de bases trocáveis com diferentes extratores, desde que haja uma boa correlação destes com a produtividade das culturas (Raij, 1994).

Outro ponto a ser observado refere-se à interpretação dos valores de CTC de um solo. Normalmente, não se consideram as particularidades do método utilizado, o que pode interferir no significado da CTC quanto à qualidade físico-química dos solos (Noble et al., 2000) e na estimação da contribuição relativa dos constituintes de solo à CTC. Um exemplo disso é calcular a CTC da argila para um grande número de solos, subtraindo a contribuição da fração orgânica à CTC, estimada em $450 \mathrm{cmol}_{\mathrm{c}} \mathrm{kg}^{-1}$ (Bennema, 1966; Camargo et al., 1987). No Sistema Brasileiro de Classificação de Solo desconsidera-se a contribuição da fração orgânica à CTC da fração argila (Embrapa, 2006).

No entanto, Bennema (1966), estudando três Latossolos, verificou uma variação de 310 a $430 \mathrm{cmol}_{\mathrm{c}} \mathrm{kg}^{-1}$ na contribuição da matéria orgânica à CTC. Nesse sentido, é de se esperar que cada tipo de solo apresente, à CTC, determinada contribuição relativa das suas frações orgânica (Bennema, 1996) e mineral (Bortoluzzi et al., 2006). Ademais, essas 
contribuições poderiam também ser diferentes caso os métodos de estimação da CTC apresentassem particularidades quanto às características químicas dos extratores, principalmente quando se utiliza apenas um extrator, como o caso do acetato de amônio e dos cloretos de hexamina Co e de K.

Sendo assim, neste trabalho, objetivou-se discutir o significado da contribuição do $\mathrm{C}$ de compostos orgânicos e da argila de um Argissolo à capacidade de troca de cátions quando diferentes métodos estão envolvidos na determinação dessa propriedade.

\section{MATERIAL E MÉTODOS}

Coletaram-se, em 2002, na área do Departamento de Solos da Universidade Federal de Santa Maria, setenta e cinco amostras, com estrutura preservada, de um Argissolo Vermelho-Amarelo distrófico abrúptico (Embrapa, 2006), representando, em triplicata, cinco camadas/horizontes $\left(\mathrm{A}_{1} 0-7,5 \mathrm{~cm}, \mathrm{~A}_{1}\right.$ 7,5-15 cm, $\mathrm{A}_{2}$, E e Bt) e cinco tipos de uso e manejo. Os manejos consistiram em campo nativo por mais de um século; vegetação arbórea espontânea por 30 anos; área de lavoura por 30 anos sob sistema de preparo convencional; área de lavoura com 15 anos sob sistema plantio direto consolidado, e lavoura por 2 anos sob plantio direto recém implantado.

O solo das amostras foi moído, tamisado em peneiras de malha de $2 \mathrm{~mm}$ e seco em temperatura ambiente, constituindo-se em terra fina seca ao ar. Posteriormente, essa fração foi submetida à caracterização química e física (Quadro 1). Para cada amostra de solo, foi determinado também o teor de argila obtido por sedimentação após dispersão em uma solução de $\mathrm{NaOH}$, segundo método descrito por Embrapa (1997). Na caracterização química, o pH foi determinado por potenciometria em presença de água e em solução com $\mathrm{CaCl}_{2}$ 0,01 mol L-1 e o teor de $\mathrm{CO}$ total (COT) pelo método da combustão a seco, utilizando um analisador elementar de $\mathrm{C} \mathrm{e} \mathrm{N}$ (Fison Carlo Erba).

Para a capacidade de troca de cátions efetiva $\left(\mathrm{CTC}_{\mathrm{E}}\right)$ e a capacidade de troca de cátions em $\mathrm{pH} 7$ $\left(\mathrm{CTC}_{7}\right)$, a caracterização foi realizada nas formas descritas a seguir.

A primeira, realizada no Laboratório de Química e Fertilidade de Solos da Universidade Federal de Santa Maria, refere-se aos métodos normalmente utilizadas no sul do Brasil e pela Comissão de Química e Fertilidade do Solo do Rio Grande do Sul e de Santa Catarina (CQFSRS/SC, 2004).

a) A capacidade de troca de cátions efetiva $\left(\mathrm{CTC}_{\mathrm{E}}\right)$ foi obtida pela soma das bases $\mathrm{Ca}^{2+}, \mathrm{Mg}^{2+} \mathrm{e} \mathrm{K}^{+}$ $\left(\mathrm{SB}_{\mathrm{KCl}+\text { Mehlich-1 }}\right)$ mais $\mathrm{Al}^{3+}\left(\mathrm{SB}+\mathrm{Al}_{\mathrm{KCl}}\right)(\mathrm{CQFSRS} / \mathrm{SC}$, 2004). Os cátions trocáveis $\mathrm{Ca}^{2+}, \mathrm{Mg}^{2+}$ e $\mathrm{Al}^{3+}$ foram deslocados com o uso da solução $\mathrm{KCl} 1 \mathrm{~mol} \mathrm{~L}^{-1}$ (Embrapa, 1997). $\mathrm{O} \mathrm{Al}^{3+}$ foi dosado por titulação ácido-base com uma solução de $\mathrm{NaOH}$ 0,0125 $\mathrm{mol} \mathrm{L}^{-1}$, enquanto o $\mathrm{Ca}^{2+} \mathrm{e} \mathrm{Mg}^{2+}$ foram dosados por espectroscopia de absorção atômica - EAA (Tedesco et al., 1995). O

Quadro 1. Atributos químicos e teor de argila obtidos pelos métodos para 75 amostras de solos apresentados em seus valores mínimos, máximos e médios, Santa Maria (RS), 2008

\begin{tabular}{|c|c|c|c|c|c|c|}
\hline \multirow{2}{*}{ Atributo } & \multicolumn{6}{|c|}{ Valor } \\
\hline & \multicolumn{2}{|c|}{ Mínimo } & \multicolumn{2}{|c|}{ Médio } & \multicolumn{2}{|c|}{ Máximo } \\
\hline COT ( $\left.\mathrm{g} \mathrm{kg}^{-1}\right)$ & \multicolumn{2}{|c|}{3,01} & \multicolumn{2}{|c|}{6,90} & \multicolumn{2}{|c|}{13,70} \\
\hline Argila (g kg-1) & \multicolumn{2}{|c|}{95,20} & \multicolumn{2}{|c|}{191,50} & \multicolumn{2}{|c|}{469,00} \\
\hline pH água & \multicolumn{2}{|c|}{3,89} & \multicolumn{2}{|c|}{4,71} & \multicolumn{2}{|c|}{6,14} \\
\hline pH SMP & \multicolumn{2}{|c|}{4,60} & \multicolumn{2}{|c|}{5,99} & \multicolumn{2}{|c|}{6,94} \\
\hline \multirow[t]{4}{*}{$\mathrm{pH} \mathrm{CaCl}{ }_{2}$} & \multicolumn{2}{|c|}{3,47} & \multicolumn{2}{|c|}{3,99} & \multicolumn{2}{|c|}{5,42} \\
\hline & \multicolumn{6}{|c|}{ Métodos descritos por } \\
\hline & \multicolumn{3}{|c|}{ CQFS RS/SC (2004) } & \multicolumn{3}{|c|}{ Afnor (1996) } \\
\hline & Mín & Méd & Máx & Mín & Méd. & Máx \\
\hline $\mathrm{Ca}\left(\mathrm{cmol}_{\mathrm{c}} \mathrm{kg}^{-1}\right)$ & 1,04 & 2,26 & 2,82 & 0,15 & 1,39 & 4,46 \\
\hline $\mathrm{Mg}\left(\mathrm{cmol}_{\mathrm{c}} \mathrm{kg}^{-1}\right)$ & 0,45 & 4,67 & 2,26 & 0,02 & 0,63 & 2,29 \\
\hline $\mathrm{Al}\left(\mathrm{cmol}_{\mathrm{c}} \mathrm{kg}^{-1}\right)$ & 0,00 & 1,43 & 4,67 & 0,00 & 1,26 & 4,53 \\
\hline $\mathrm{K}\left(\mathrm{cmol}_{\mathrm{c}} \mathrm{kg}^{-1}\right)$ & 0,00 & 0,09 & 0,30 & 0,01 & 0,11 & 0,35 \\
\hline $\mathrm{SB}\left(\mathrm{cmol}_{\mathrm{c}} \mathrm{kg}^{-1}\right)$ & 1,61 & 2,79 & 5,38 & 0,37 & 2,16 & 7,03 \\
\hline $\mathrm{CTC}_{\mathrm{E}}\left(\mathrm{cmol}_{\mathrm{c}} \mathrm{kg}^{-1}\right)$ & 2,44 & 4,22 & 7,50 & 1,40 & 4,04 & 7,70 \\
\hline $\mathrm{CTC}_{7}\left(\mathrm{cmol}_{\mathrm{c}} \mathrm{kg}^{-1}\right)$ & 4,20 & 8,52 & 24,80 & 2,80 & 5,29 & 10,03 \\
\hline
\end{tabular}

COT: carbono orgânico total determinado por combustão úmida; argila determinada seguindo Embrapa (1997); pH determinado em presença de água, solução tampão $\mathrm{SMP}$ e salina de $\mathrm{CaCl}_{2} 0,01 \mathrm{~mol} \mathrm{~L}^{-1}$; $\mathrm{SB}$ : soma de bases; $\mathrm{CTC}_{\mathrm{E}}$ e $\mathrm{CTC}_{7}$ : capacidade de troca de cátions efetiva e em pH 7. CQFSRS/SC (2004) $\mathrm{Al}$, Ca e $\mathrm{Mg}$ extraídos por $\mathrm{KCl} 1 \mathrm{~mol} \mathrm{~L}^{-1}$ e $\mathrm{K}_{\mathrm{E}}$ por Mehlich-1; CTC $\mathrm{E}_{\mathrm{E}}$ e CTC determinados pela soma de bases mais $\mathrm{Al} \mathrm{e} \mathrm{H}+\mathrm{Al}$, respectivamente. Afnor (1996) $\mathrm{Al}$ extraído por $\mathrm{KCl}$ e $\mathrm{Ca}$, $\mathrm{Mg}$ e $\mathrm{K}$ por cloreto de hexamina Co; $\mathrm{CTC}_{\mathrm{E}}$ e $\mathrm{CTC}_{7}$ determinadas por cloreto de hexamina Co e por acetato de $\mathrm{NH}_{4}^{+}$, respectivamente. 
$\mathrm{K}^{+}$trocável foi deslocado pela solução Mehlich-1 ( $\mathrm{HCl}$ $0,05 \mathrm{~mol} \mathrm{~L}^{-1}+\mathrm{H}_{2} \mathrm{SO}_{4} 0,0125 \mathrm{~mol} \mathrm{~L}^{-1}$ ) e medido por espectroscopia de emissão de chama.

b) A capacidade de troca de cátions em $\mathrm{pH} 7\left(\mathrm{CTC}_{7}\right)$ foi estimada pela soma de bases mais $\mathrm{H}+\mathrm{Al}$ $\left(\mathrm{SB}+\mathrm{H}+\mathrm{Al}_{\mathrm{SMP}}\right.$ ), ao passo que os valores de $\mathrm{H}+\mathrm{Al}$ foram estimados pela seguinte equação, proposta por Kaminski et al. (2002) e adotada pela CQFSRS/SC (2004):

$$
\mathrm{H}+\mathrm{Al}=\frac{e^{10,665-1,1483^{*} p H S M P}}{10}
$$

em que o teor no solo de $\mathrm{H}+\mathrm{Al}$ é estimado em $\mathrm{cmol}_{\mathrm{c}} \mathrm{kg}^{-1}$ e o índice SMP é a medida do $\mathrm{pH}$ de equilíbrio do solo com a solução SMP (Shoemaker et al., 1961). A solução SMP tem uma concentração de $1,78 \mathrm{~mol} \mathrm{~L}^{-1}$ tamponada em $\mathrm{pH} 7,5$, composta por trietanolamina, paranitrofenol, $\mathrm{K}_{2} \mathrm{CrO}_{4}$, $\mathrm{Ca}\left(\mathrm{CH}_{3} \mathrm{COO}\right)_{2}$ e $\mathrm{CaCl}_{2} \cdot 2 \mathrm{H}_{2} \mathrm{O}$ (Tedesco et al., 1995).

A segunda etapa, realizada no Laboratório de Química do Institut National de la Recherche Agronomique em Arras, França, seguiu os métodos preconizados pela Associação Francesa de Normas Técnicas-AFNOR (Afnor, 1996), compreendendo as seguintes determinações: (a) a $\mathrm{CTC}_{\mathrm{E}}$ foi estimada pelo método do cloreto de hexamina Co a 0,01666 mol L-1 $\left(\right.$ CTC $_{\mathrm{E}}$ Cohex) (Afnor, 1996; Ciesielski \& Sterckeman, 1997); os cátions $\mathrm{Ca}^{2+}, \mathrm{Mg}^{2+}$ e K ${ }^{+}$foram extraídos com cloreto de hexamina $\mathrm{Co}$, sendo os dois primeiros dosados por EAA e o $\mathrm{K}^{+}$por espectrometria de emissão de chama (Orsini \& Remy, 1976; Ciesielski \& Sterckeman, 1997); a soma de bases foi conseguida somando-se os teores de cátions $\mathrm{Ca}^{2+}, \mathrm{Mg}^{2+}$ e $\mathrm{K}^{+}$ extraídos por cloreto de hexamina Co (SB Cohex) (Afnor, 1996). (b) $\mathrm{A} \mathrm{CTC}_{7}$ foi determinada por troca com acetato de $\mathrm{NH}_{4}{ }^{+} 1 \mathrm{~mol} \mathrm{~L}^{-1}$ (CTC Metson) (Metson,
1956). Em resumo, por percolação da solução $\mathrm{NH}_{4}{ }^{+}$ saturam-se os sítios de carga do solo, concomitante à extração dos cátions. Para a determinação da CTC, a amostra é lavada com etanol, retirando-se o excesso de reagente, e seca ao ar; o $\mathrm{NH}_{4}{ }^{+}$é então medido por espectrocolorimetria após ser deslocado com uma solução de cloreto de sódio $1 \mathrm{~mol} \mathrm{~L}^{-1}$.

Os teores de $\mathrm{Al}^{3+}$ trocável foram extraídos por $\mathrm{KCl}$ $1 \mathrm{~mol} \mathrm{~L}^{-1} \mathrm{em}$ ambos os métodos, e os de $\mathrm{Na}^{+}$, por tenderem a zero em todas as amostras, não foram considerados no cálculo da soma de bases. Vale ressaltar que cada método usa diferentes extratores, com diferentes potenciais tampão e forças iônicas. Os valores mínimos, médios e máximos obtidos pelas análises químicas do solo estão também apresentados no quadro 1.

Os valores de cátions e da $\mathrm{CTC}_{\mathrm{E}}$ e $\mathrm{CTC}_{7}$ foram relacionados entre si por meio do teste de correlação de Pearson e por regressões lineares. Compararamse os métodos por meio dos coeficientes lineares e angulares das equações de regressão linear, segundo metodologia de Miller \& Miller (1993), utilizada por Valladares et al. (2001). A hipótese de nulidade referiuse ao coeficiente angular que não diferiria de 1,0 e ao coeficiente linear que não diferiria de zero. Intervalos de confiança a $p<0,01$ foram obtidos com o uso do pacote estatístico Excel ${ }^{\circledR}$ e serviram para testar essas hipóteses, estando sumarizados no quadro 2. Assim, considerar-se-ão os métodos diferentes entre si quando os coeficientes angulares e lineares das equações de regressão e seus correspondentes limites calculados não englobarem o valor 0 e 1 , respectivamente. Quanto à contribuição dos constituintes à $\mathrm{CTC}_{7}$, utilizar-se-á o método estatístico gráfico proposto por Helling et al. (1964) e adotado por Bennema (1966). A interpretação das suas equações seguirá como descrita anteriormente.

Quadro 2. Estimativa do intervalo de confiança dos coeficientes angulares (b) e lineares (a) de regressões lineares $(\hat{y}=\mathbf{a}+\mathbf{b x})$, onde $(\hat{y})$ refere-se aos métodos preconizados pela CQFSRS/SC (2004) e (x) a Afnor (1996) para 75 de amostras de um Argissolo do sul do Brasil

\begin{tabular}{|c|c|c|c|c|}
\hline \multirow{2}{*}{ Atributo: $(\hat{\mathrm{y}})$ CQFSRS/SC (2004) vs (x) Afnor (1996) } & \multicolumn{2}{|c|}{$\mathbf{a}^{(1)}$} & \multicolumn{2}{|c|}{$\mathbf{b}^{(1)}$} \\
\hline & Mínimo & Máximo & Mínimo & Máximo \\
\hline $\mathrm{Ca}: \mathrm{KCl}$ us Cohex & 0,9547 & 1,0504 & 0,4220 & 0,4779 \\
\hline $\mathrm{Mg}: \mathrm{KCl}$ vs Cohex & 0,3548 & 0,4353 & 1,0039 & 1,1077 \\
\hline K: Mehlich-1 vs Cohex & $-0,0442$ & $-0,0178$ & 1,0166 & 1,2064 \\
\hline SB: $\mathrm{KCl}+$ Mehlich -1 vs Cohex & 1,2150 & 1,6239 & 0,5740 & 0,7332 \\
\hline $\mathrm{CTC}_{\mathrm{E}}:\left(\mathrm{SB}+\mathrm{Al}_{\mathrm{KCl}}\right)$ vs Cohex & 1,0985 & 2,1559 & 0,5218 & 0,7609 \\
\hline $\mathrm{CTC}_{7}:\left(\mathrm{CTC}_{\mathrm{E}}+\mathrm{H}+\mathrm{Al}_{\mathrm{SMP}}\right)$ vs Metson & $-4,1781$ & 0,5168 & 1,5455 & 2,3677 \\
\hline $\mathrm{CTC}_{7}:$ Metson vs CTC $_{\mathrm{E}}$ Cohex & 0,2061 & 1,5225 & 0,9462 & 1,2437 \\
\hline $\mathrm{CTC}_{7}\left(\mathrm{CTC}_{\mathrm{E}}+\mathrm{H}+\mathrm{Al}_{\mathrm{SMP}}\right)$ vs $\mathrm{CTC}_{\mathrm{E}}\left(\mathrm{SB}+\mathrm{Al}_{\mathrm{KCl}}\right)$ & $-7,0234$ & $-0,3711$ & 2,1443 & 3,6464 \\
\hline
\end{tabular}

${ }^{(1)}$ Significativo a $1 \%$ pelo teste $\mathrm{F}$. 


\section{RESULTADOS E DISCUSSÃO}

\section{Comparação de métodos na extração de cátions e na estimação da CTC}

$\mathrm{O}$ solo das amostras apresentou amplitude nos teores de argila, variando de 95,2 a $469 \mathrm{~g} \mathrm{~kg}^{-1}$, COT de 3,01 a 13,70 $\mathrm{g} \mathrm{kg}^{-1}$, e das CTCs de 1,4 a $24,80 \mathrm{cmol}_{\mathrm{c}} \mathrm{kg}^{-1}$. Os teores de cátions obtidos pelos métodos Cohex e soma de bases (SB), em geral, apresentaram boa correlação entre si, acima de 0,95 e suas equações de regressão apontam coeficientes de determinação superiores a $R^{2}=0,93$ (Figura 1). Isso demonstra que os extratores químicos, mesmo utilizando diferentes cátions e concentrações, são capazes de discriminar variações nos teores de cátions do solo. Contudo, no quadro 2, observam-se discrepâncias quanto aos coeficientes angulares e lineares em relação à linha $\mathrm{Y}=\mathrm{X}$.

Quando a extração de Ca com Cohex é nula, o extrator $\mathrm{KCl}$ extrai $1 \mathrm{cmol}_{\mathrm{c}} \mathrm{kg}^{-1},(\hat{\mathrm{y}}=1+0,45 \mathrm{x})$, mas este último apresenta menor capacidade de extração, coeficiente angular de 0,45, diferente de 1,0 (Quadro 2). Como a literatura não menciona correlações entre $\mathrm{KCl}$ e Cohex, pode-se inferir que o primeiro extrator desloca mais Ca trocável quando este se apresenta em baixos teores no solo. $\mathrm{O}$ ponto de interseção entre a reta $\mathrm{Y}=\mathrm{X}$ e ao da regressão é próximo de $2 \mathrm{cmol}_{\mathrm{c}} \mathrm{kg}^{-1}$, em torno desse valor a extração dos métodos não difere (Quadro 2).

Para o Mg, o extrator $\mathrm{KCl}$ extrai sistematicamente mais $\mathrm{Mg}$ que o Cohex $(\hat{\mathrm{y}}=0,4+1,06 \mathrm{x})$, porque os coeficientes linear de 0,4 e angular de 1,06 diferem de zero e de 1,0, respectivamente (Quadro 2).

A capacidade de extração do extrator Mehlich-1 e Cohex é também diferente para o K trocável $(\hat{y}=-0,03$ $+1,11 \mathrm{x}$ ), visto que os teores de $\mathrm{K}$ extraídos pelo Mehlich-1 foram $11 \%$ maiores (coeficiente angular de 1,11) que os obtidos pelo Cohex (Quadro 2). Apesar de os extratores apresentarem diferentes capacidades de extração de cátions do solo, representados pelo coeficiente angular diferente de 1 , eles têm alto grau de relacionamento, $R^{2}>0,93$ (Figura 1). Isso é importante porque permite a transformação dos valores quando obtidos por um ou outro método (Raij \& Kupper, 1966; Jönsson et al., 2002). Já o fato de os coeficientes lineares serem discrepantes entre os
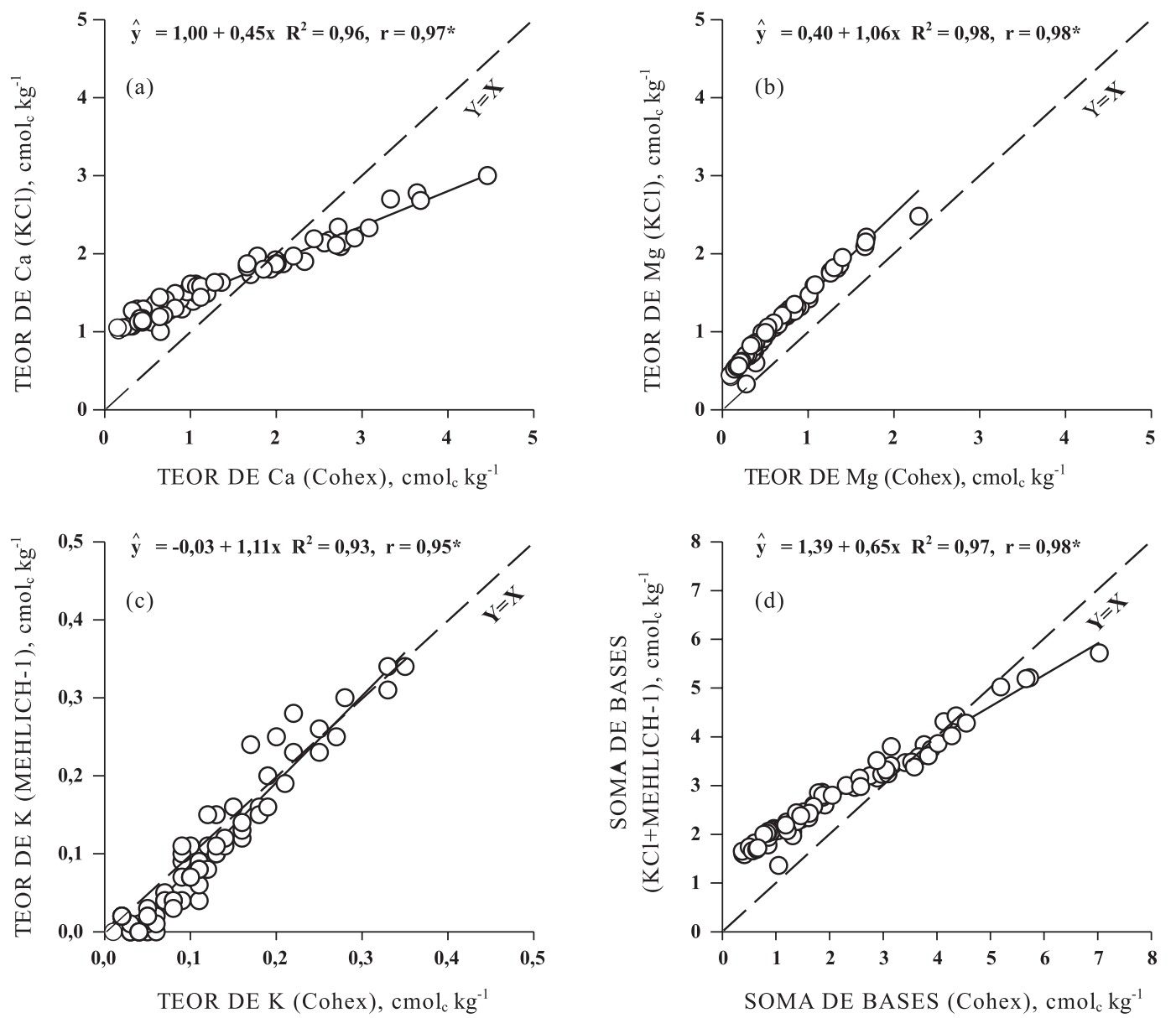

Figura 1. Relação entre valores de cátions e soma de bases obtidos por diferentes métodos em 75 amostras de um Argissolo subtropical. 
métodos indica que fatores intrínsecos a cada método são atuantes por exemplo a força iônica.

A resposta conjunta da extração dos cátions $\mathrm{Ca}$, Mg e K entre os dois métodos (Figuras 1abc) é refletida em seu somatório (Figura 1d). A equação linear que representa essa relação $\hat{y}=1,39+0,65 x$, apresenta alto coeficiente de determinação, $R^{2}=0,97$. Percebese que, apesar das diferenças encontradas entre os métodos na extração de $\mathrm{Mg}$ e $\mathrm{K}$, foi a extração do $\mathrm{Ca}$ que influenciou a relação entre a soma de bases entre os métodos. Isso porque a extração de $\mathrm{Ca}$ pelo $\mathrm{KCl}$ apresentou uma amplitude que variou de 1,0 a $2,8 \mathrm{cmol}_{\mathrm{c}} \mathrm{kg}^{-1}$, enquanto o extrator Cohex apresentou uma amplitude mais larga de 0,15 a 4,46 $\mathrm{cmol}_{\mathrm{c}} \mathrm{kg}^{-1}$ (Quadro 1). É provável, segundo Raij \& Kupper (1966), que a valência do cátion extrator $\left(\mathrm{K}^{+}\right.$do $\left.\mathrm{KCl}\right) \mathrm{e}$ a força iônica da solução $\mathrm{KCl}$ de $1 \mathrm{~mol} \mathrm{~L}^{-1}$ tenham interferido na extração de $\mathrm{Ca}$, o que resultou, neste estudo, em menor eficiência em deslocar o elemento dos sítios de troca. Já o método Cohex usa uma solução menos concentrada $\left(0,01666 \mathrm{~mol} \mathrm{~L}^{-1}\right)$, e é citado por Ciesielski \& Sterckeman (1997) como similar ao método que usa cloreto de bário $\left(\mathrm{BaCl}_{2}\right)$.

A equação que representa a relação entre os valores de $\mathrm{CTC}_{\mathrm{E}}$ obtidos pelo método $\left(\mathrm{SB}+\mathrm{Al}_{\mathrm{KCl}}\right)$ e pelo método (Cohex) é $\hat{y}=1,63+0,64 x\left(R^{2}=0,73\right)$ (Figura 2a). A relação indica que o método $\left(\mathrm{SB}+\mathrm{Al}_{\mathrm{KCl}}\right)$ parte de valores de $\mathrm{CTC}_{\mathrm{E}}$ de $1,63 \mathrm{cmol}_{\mathrm{c}} \mathrm{kg}^{-1}$ quando o Cohex é nulo. Porém, o Cohex apresenta uma capacidade de extração maior comparado ao método $\mathrm{SB}+\mathrm{Al}_{\mathrm{KCl}}$ utilizado pela CQFSRS/SC (2004). Isto se deve provavelmente ao comportamento dos extratores principalmente na extração de $\mathrm{Ca}$, visto que se trata do cátion que contribuiu em maior quantidade à CTC.

Para a $\mathrm{CTC}_{7}$, em que ambos os métodos $\left(\mathrm{SB}+\mathrm{H}+\mathrm{Al}_{\mathrm{SMP}}\right)$ e Metson usam soluções tamponadas, tem-se a relação entre eles expressa pela equação $\hat{y}=-1,83+1,66 x$, com coeficiente de determinação de $R^{2}=0,68$ (Figura $2 b$ ). O coeficiente linear (a) não diferiu de zero (Quadro 2), mas o coeficiente angular é diferente de 1. Segundo Ikawa (1978) e Duriez et al. (1988), o acetato de amônio tem capacidade equivalente de extração em relação ao extrator $\mathrm{KCl}+$ Mehlich-1. Assim, dois prováveis fatores podem ter atuado para explicar esse fato (Ciesielski \& Sterckeman, 1997). O primeiro diz respeito ao maior $\mathrm{pH}$ de tamponamento da solução SMP (pH 7,5) comparado ao acetato de amônio (pH 7,0). Percebe-se, em função disso, que o extrato $\mathrm{NH}_{4}{ }^{+}$(método Metson) restringe os valores de $\mathrm{CTC}_{7}$ a cerca de $10 \mathrm{cmol}_{\mathrm{c}} \mathrm{kg}^{-1}$, enquanto o método que usa SMP apresenta valores amplos, chegando a $30 \mathrm{cmol}_{\mathrm{c}} \mathrm{kg}^{-1}$. O segundo fator está relacionado à característica do cátion índice na solução extratora. $\mathrm{O}$ fato de o cátion indicador ser único, como o $\mathrm{NH}_{4}{ }^{+}$no método Metson, e não uma mistura de extratores como no método preconizado pela CQFS-RS/SC (2004), pode ser responsável por subestimações na medida da $\mathrm{CTC}_{7}$ pelo Metson. Isso porque o $\mathrm{NH}_{4}{ }^{+}$tem seu raio iônico similar ao do $\mathrm{K}^{+}$hidratado e, por consequência, é adsorvido à cavidade siloxana dos argilominerais 2:1 (Chaminade, 1971; Raij \& Kupper, 1966; Chappell \& Evangelou, 2000). Essa interpretação é plausível, visto que nesse tipo de solo ocorrem argilominerais do tipo ilita-vermiculita, esmectita e vermiculita com hidroxiAl entrecamada, todos com potencial de fixar $K$ entrecamadas (Bortoluzzi et al., 2005, 2008a,b).

A figura 3a mostra a relação entre os valores de $\mathrm{CTC}_{\mathrm{E}}$ (Cohex) e $\mathrm{CTC}_{7}$ (Metson). A equação linear de regressão que expressa essa relação é $\hat{y}=0,86+1,09 x$ $\left(R^{2}=0,84\right)$. Os valores de $\mathrm{CTC}_{7}$ apresentam incrementos, em média, de $9 \%$ nos valores de $\mathrm{CTC}_{\mathrm{E}}$, novamente mostrando que o acetato de amônio não tem capacidade de extração de hidrogênio ligado covalentemente aos grupos funcionais com ponto de carga zero mais elevado (Charlet \& Schlegel, 1999). Já a relação entre o método que usa a solução SMP para estimar a $\mathrm{CTC}_{7}\left(\mathrm{CTC}_{\mathrm{E}}+\mathrm{H}+\mathrm{Al}_{\mathrm{SMP}}\right)$ e a $\mathrm{CTC}_{\mathrm{E}}$ $\left(\mathrm{SB}+\mathrm{Al}_{\mathrm{KCl}}\right)$ a regressão é expressa pela equação $\hat{\mathrm{y}}=$ $-3,69+2,89 x\left(R^{2}=0,59\right)$ (Figura 3b). Isso indica que as amplitudes de extração são diferentes entre os valores de $\mathrm{CTC}_{7}$, sendo mais elevados (máximo chegando a $25 \mathrm{cmol}_{\mathrm{c}} \mathrm{kg}^{-1}$ ) do que os valores da $\mathrm{CTC}_{\mathrm{E}}$,
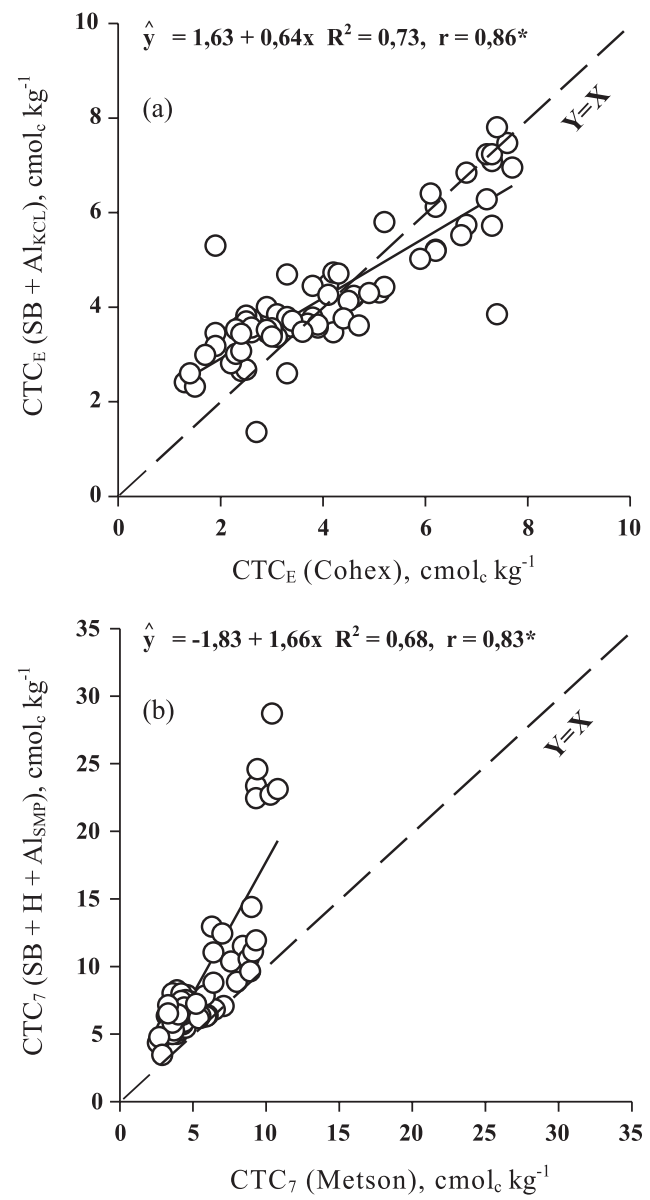

Figura 2. Relação entre (a) CTC efetiva e (b) CTC $_{7}$ obtidas por diferentes métodos em 75 amostras de um Argissolo subtropical. 

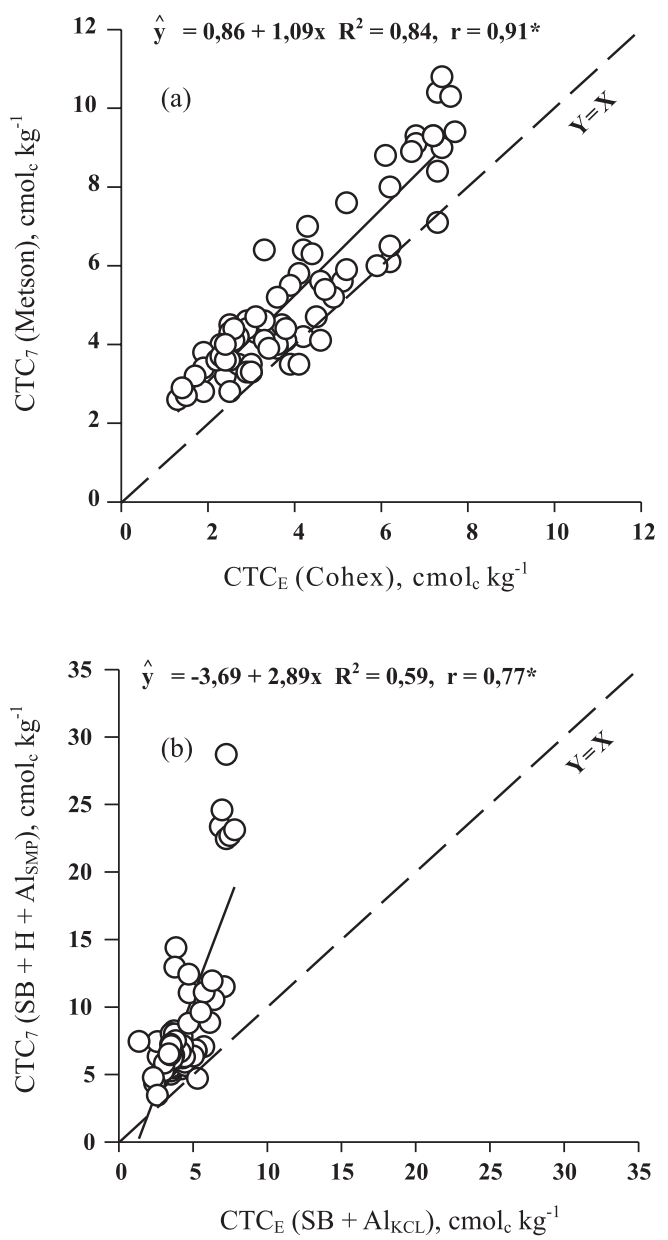

Figura 3. Relação entre $\mathrm{CTC}_{7}$ e efetiva para diferentes métodos de estimação a) segundo Afnor (1996) e b) segundo CQFSRS/SC (2004).

demonstrando que a acidez potencial é mais bem estimada pela solução SMP (Shoemaker et al., 1961) do que com o acetato de amônio.

\section{Contribuição dos constituintes à capacidade de troca de cátions}

Observa-se que os métodos apresentados estimam a CTC com magnitudes distintas. A premissa básica para a CTC é de que esta demonstra o equilíbrio químico soluto:sorvente a um dado estado químico do solo (Anderson \& Sposito, 1992). Esses autores preconizam que os cátions trocáveis estão em igualdade estequiométrica às cargas elétricas permanentes e variáveis dos constituintes orgânicos e minerais do solo, sendo regidos pelos fenômenos de adsorção. Assim, ao ser relacionado os valores de CTC com os de pH e com teores de $\mathrm{CO}$ total do solo esperamse altos graus de relacionamento. Contudo, frequentemente isso não ocorre, como verificado por Souza et al. (2007), devido principalmente à variação na mineralogia e no tipo e conteúdo de matéria orgânica entre os horizontes de solo (Klamt \& van Reeuwijk, 2000). Uma maneira de eliminar o problema é subtrair a interferência do $\mathrm{pH}$, como proposto por Katou (2002). Outra maneira é associar a contribuição conjunta à CTC dos dois constituintes (argila e COT) sem levar em conta os valores medidos de $\mathrm{pH}$, isto porque eles são integrantes dos valores de $\mathrm{CTC}_{\mathrm{E}}$ e influenciam o tamponamento com $\mathrm{H}+\mathrm{Al}$.

Assim, na figura 4, tem-se a contribuição da argila e do $\mathrm{C}$ à $\mathrm{CTC}_{7}$ estimada pelos métodos preconizados pela CQFSRS/SC (2004) e Afnor (1996). Observa-se que, para a $\mathrm{CTC}_{7}$ estimada pelo método $\left(\mathrm{SB}+\mathrm{H}+\mathrm{Al}_{\mathrm{SMP}}\right)$, a equação linear múltipla é expressa como $\hat{\mathrm{y}}=0,023$ Argila $+0,399 \mathrm{COT}$, com coeficiente de determinação de $R^{2}=0,86$. Assim, a contribuição das frações à $\mathrm{CTC}_{7}$ é de $23 \mathrm{cmol}_{\mathrm{c}} \mathrm{kg}^{-1} \mathrm{e}$ $399 \mathrm{cmol}_{\mathrm{c}} \mathrm{kg}^{-1}$, respectivamente para a argila e o COT. Para a $\mathrm{CTC}_{7}$ (Metson), a relação é expressa pela equação linear múltipla $\hat{y}=0,019$ Argila $+0,256 \mathrm{COT}$, com coeficiente de determinação de $R^{2}=0,97$. Portanto, a contribuição da fração argila (teor de argila) à $\mathrm{CTC}_{7}$ é de $19 \mathrm{cmol}_{\mathrm{c}} \mathrm{kg}^{-1}$ de argila, enquanto o COT contribui com $256 \mathrm{cmol}_{\mathrm{c}} \mathrm{kg}^{-1}$.

Neste estudo, a contribuição da fração orgânica à $\mathrm{CTC}_{7}(\mathrm{SB}+\mathrm{H}+\mathrm{Al})$ foi superior ao encontrado por Bayer \& Mielniczuk (1997), que é de $314 \mathrm{cmol}_{\mathrm{c}} \mathrm{kg}^{-1}$ de COT. Vale notar que ambos os trabalhos utilizaram os mesmos métodos, assim como a origem das amostras representam diferentes manejos e profundidades de solos. No entanto, a contribuição COT à CTC Metson é de $256 \mathrm{cmol}_{\mathrm{c}} \mathrm{kg}^{-1}$, valor menor que aquele encontrado pelo método $\mathrm{CTC}_{7}$ ( $\mathrm{SB}+\mathrm{H}+\mathrm{Al}_{\mathrm{SMP}}$ ), que é de $399 \mathrm{cmol}_{\mathrm{c}} \mathrm{kg}^{-1}$. Isto é devido às diferentes características de extração dos métodos utilizados. Por exemplo, o acetato de amônio tem menor capacidade de extração de cátions em solos que tem teores mais baixos de matéria orgânica (Skinner et al., 2001).

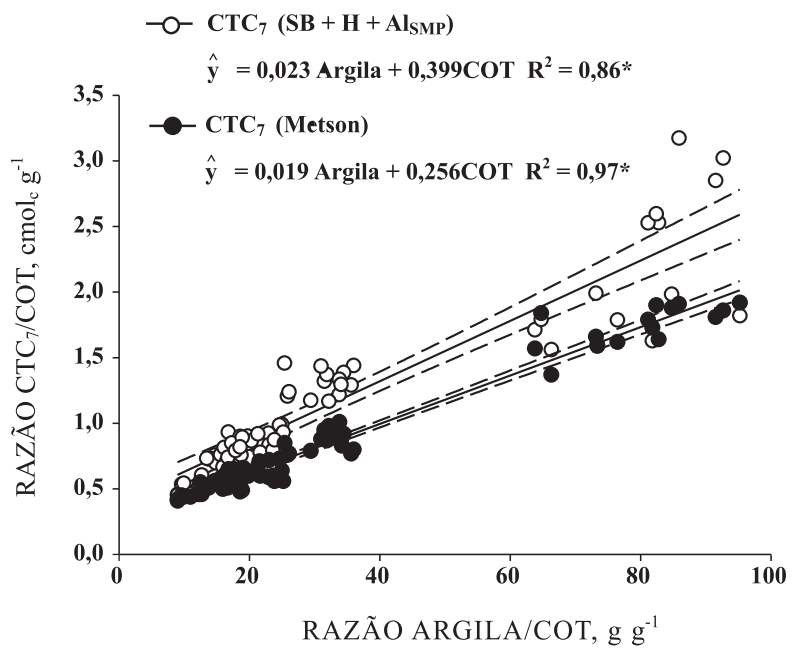

Figura 4. Contribuição da fração de argila e de carbono orgânico total (COT) à capacidade de troca de cátions potencial obtidas pelos métodos preconizados pela CQFSRS/SC (2004) e pela (Afnor, 1996). As linhas pontilhadas são os intervalos de confiança $(p<0,01)$. 
Ao confrontar a contribuição da fração orgânica desse solo a Latossolos (de 310 a $430 \mathrm{cmol}_{\mathrm{c}} \mathrm{kg}^{-1}$ ) estudados por Bennema (1966), percebe-se que a contribuição fica subestimada quando usado o método do acetato de amônio e superestimada para o método que usa a solução SMP. Para os horizontes B, a CTC da argila é importante como propriedade diagnóstica - atividade da argila (Camargo et al., 1987, Embrapa, 2006). Para esse fim, Bennema (1966) e Camargo et al. (1987) usaram uma equação em que a contribuição do carbono à CTC é de $450 \mathrm{cmol}_{\mathrm{c}} \mathrm{kg}^{-1}$, valor acima daquele encontrado neste estudo. Esses resultados coadunam as observações de Klamt \& Reeuwijk (2000), alertando para o fato que a estimativa da contribuição do carbono à CTC deveria ser usada para cada tipo de solo senão para cada perfil.

Quanto à contribuição da fração argila à CTC, percebe-se que o valor de $23 \mathrm{cmol}_{\mathrm{c}} \mathrm{kg}^{-1}$ é muito superior às cargas da caulinita, de 1 a $10 \mathrm{cmol}_{\mathrm{c}} \mathrm{kg}^{-1}$ (Parfitt et al., 1995; Ma \& Eggleton, 1999). A explicação é que a fração argila desse Argissolo apresenta também cargas permanentes oriundas dos argilominerais do tipo 2:1 vermiculita-ilita, esmectita e caulinita-esmectita como verificada por Bortoluzzi et al. (2008a,b).

Em geral, os métodos foram capazes de estimar as variações de concentração dos cátions adsorvidos aos sítios de troca das amostras de solo, e, por conseguinte, estimam satisfatoriamente a sua CTC. No entanto, quando os métodos são confrontados, observam-se peculiaridades nas magnitudes da CTC estimada, o que pode afetar a interpretação de propriedades de solos como a contribuição das frações orgânica e mineral à CTC.

\section{CONCLUSÕES}

1. A capacidade de troca de cátions estimada pelos métodos baseados na extração com solução Mehlich1 , cloreto de hexamina cobalto, acetato de amônio e SMP relacionam-se entre si com alto grau de correlação, apesar das diferentes magnitudes de extração.

2. A contribuição da argila e do carbono orgânico total do solo à $\mathrm{CTC}_{7}$ é dependente do método utilizado para sua estimação, sendo respectivamente $23 \mathrm{e}$ $399 \mathrm{cmol}_{\mathrm{c}} \mathrm{kg}^{-1}$ para a CTC 7 SMP e 19 e $256 \mathrm{cmol}_{\mathrm{c}} \mathrm{kg}^{-1}$ para a $\mathrm{CTC}_{7}$ Metson. As contribuições dos constituintes de solo à capacidade de troca de cátion são subestimadas para o método Metson, que usa o acetato de amônio comparado ao método que usa a solução SMP, utilizado no sul do Brasil.

\section{AGRADECIMENTOS}

Ao pesquisador do INRA, Dr. Daniel Tessier, pela viabilidade das análises segundo o protocolo Afnor realizada em Arras, França.

\section{LITERATURA CITADA}

ANDERSON, S. \& SPOSITO, G. Proton surface-charge density in soils with structural and $\mathrm{pH}$-dependent charges. Soil Sci. Soc. Am. J., 56:1437-1443, 1992.

ASSOCIATION FRANÇAISE DES NORMES TECHNIQUES - AFNOR. Qualité des sols: Recueil des normes françaises. 3.ed. Paris la Défense, 1996. 534p.

BARROW, N.J. The four laws of soil chemistry: The Leeper lecture 1998. Austr. J. Soil. Res., 37:787-829, 1999.

BAYER, C. \& MIELNICZUK, J. Características químicas do solo afetadas por métodos de preparo e sistemas de cultura. R. Bras. Ci. Solo, 21:105-112, 1997.

BENNEMA, J. The calculation of CEC for 100 grams clay (CEC 100) with correction for organic carbon. In: Report to the government of Brazil on classification of Brazilian soils. Rome, FAO, 1966. 83p. (FAO, EPTA, 2197)

BORTOLUZZI, E.C.; RHEINHEIMER, D.S.; KAMINSKI, J.; GATIBONI, L. \& TESSIER, D. Alterações na mineralogia de um Argissolo do Rio Grande do Sul submetido à fertilização potássica. R. Bras. Ci. Solo, 29:327-335, 2005.

BORTOLUZZI, E.C.; TESSIER, D.; RHEINHEIMER, D.S. \& JULIEN, J.L. The cation exchange capacity of a sandy soil in southern Brazil: An estimation of permanent charge and $\mathrm{pH}$-dependent charges. Eur. J. Soil Sci., 57:356-364, 2006.

BORTOLUZZI, E.C.; PERNES, M. \& TESSIER. D. Mineralogia de partículas envolvidas na formação de gradiente textural em um Argissolo subtropical. R. Bras. Ci. Solo, 32:9971007, 2008a.

BORTOLUZZI, E.C.; VELDE, B.; PERNES, M.; DUR, J.C. \& TESSIER. D. Vermiculite with hydroxy-aluminium interlayer and kaolinite formation in a subtropical sandy soil from south Brazil. Clay Miner., 43:155-163, 2008b.

CAMARGO, M.N.; KLAMT, E. \& KAUFFMAN, J.H. Sistema brasileiro de classificação de solos. B. Inf. SBCS, 12:1133, 1987.

COMISSÃO DE QUÍMICA E FERTILIDADE DE SOLO DO RIO GRANDE DO SUL E DE SANTA CATARINA CQFSRS/SC. Recomendações de adubação e de calagem para os estados do RS e SC. 3.ed. Passo Fundo, Comissão de Fertilidade do Solo - RS/SC, SBCS-Núcleo Regional Sul, 2004. 400p.

CHAPPELL, M.A. \& EVANGELOU, V.P. Influence of added $\mathrm{K}^{+}$on ammonium selectivity/mobility by soils with vermiculate behavior. Soil Sci., 165:858-868, 2000.

CHAMINADE, R. Recherches sur la rétrogradation de l'ion ammonium. Ann. Agron., 22:343-361, 1971.

CHARLET, L. \& SCHLEGEL, M.L. La capacité d'échange des sols. Structure et charges à l'interface eau/particule. CR. Acad. Fr., 85:7-24, 1999.

CIESIELSKI, H. \& STERCKEMAN, T. A comparison between three methods for the determination of cation exchange capacity and exchangeable cations in soils. Agronomie, 17:9-16, 1997. 
DURIEZ, M.A.M.; MELO, M.E.C.C.M.; JOHAS, R.A.L. \& ARAUJO, N.S. Apparent ECEC in some brazilian soils with variable charges determined using three different extractors. In: INTERNATIONAL SOIL CLASSIFICATION WORKSHOP, 8., Rio de Janeiro, 1986. Proceedings. Rio de Janeiro, Embrapa-SNLCS, 1988. p.49-55.

EMPRESA BRASILEIRA DE PESQUISA AGROPECUÁRIA EMBRAPA. Centro Nacional de Pesquisa de Solos. Manual de métodos de análise do solo. 2.ed. Rio de Janeiro, 1997.

EMPRESA BRASILEIRA DE PESQUISA AGROPECUÁRIA EMBRAPA. Centro Nacional de Pesquisa de Solos. Sistema brasileiro de classificação de solos. 2.ed. Brasília, 2006. 306p.

GATIBONI, L.C.; KAMINSKI, J.; RHEINHEIMER, D.S. \& FLORES, J.P.C. Biodisponibilidade de formas de fósforo acumuladas em solo sob sistema plantio direto. R. Bras. Ci. Solo, 31:691-699, 2007.

HELLING, C.S.; CHESTERS, G. \& COREY, R.B. Contribution of organic matter and clay to soil cation-exchange capacity as affected by the $\mathrm{pH}$ of the saturating solution. Soil Sci. Soc. Am. Proc., 28:517-520, 1964.

IKAWA, H. Correlation of selected data for some brazilian soils provided by Brazil and SCS-USDA laboratories. In: INTERNATIONAL SOIL CLASSIFICATION WORKSHOP, 1., Rio de Janeiro, 1977. Proceedings. Rio de Janeiro, Embrapa-SNLCS, 1978. p.338-353.

JÖNSSON, U.; ROSENGREN, U.; NIHLGÅRD, B. \& THELIN, G. A comparative study of two methods for determination of $\mathrm{pH}$, exchangeable base cations, and aluminum. Comm. Soil Sci. Plant Anal., 33:3809-3824, 2002.

KAMINSKI, J.; GATIBONI, L.C.; RHEINHEIMER, D.S.; MARTINS, J.R.; SANTOS, E.J.S. \& TISSOT, C.A. Estimativa da acidez potencial em solo e sua implicação no cálculo da necessidade de calagem. R. Bras. Ci. Solo, 26:1107-1113, 2002 .

KAMINSKI, J.; BRUNETTO, G.; MOTERLE, D.F. \& RHEINHEIMER, D.S. Depleção de formas de potássio do solo afetada por cultivos sucessivos. R. Bras. Ci. Solo, 31:1003-1010, 2007

KATOU, H. A pH-dependence implicit formulation of cationand anion-exchange capacities of variable-charge soils. Soil Sci. Soc. Am. J., 66:1218-1224, 2002.

KLAMT, E. \& van REEUWIJK, L.P. Evaluation of morfological, physical and chemical characteristics of ferralsols and related soil. R. Bras. Ci. Solo, 24:573:587, 2000.

MA, C. \& EGGLETON, R.A. Cation exchange capacity of kaolinite. Clays Clay Miner., 47:174-180, 1999.

METSON, A.J. Methods of chemical analysis for survey samples. N.Z Soil Bur Bull., n.12, 208p. 1956.
MILLER, J.C. \& MILLER, J.N. Statistics for analytical chemistry. 3.ed. Chichester, Ellis Horwood, 1993. p.120124.

NOBLE, A.D.; GILLMAN, G.P. \& RUAYSOONGNERN, S. A cation exchange index for assessing degradation of acid soil by further acidification under permanent agriculture in the tropics. Eur. J. Soil Sci., 51:233-243, 2000.

ORSINI, L. \& REMY, J.C. Utilisation du chlorure de cobalti hexamine pour la détermination simultanée de la capacité d'échange et des bases échangeables des sols. B. l'AFES, Sci. Sol, 4:269-275, 1976.

PARFITT, R.L.; GILTRAP, D.J. \& WHITTON, J.S. Contribution of organic matter and clay minerals to the cation exchange capacity of soils. Comm. Soil Sci. Plant. Anal., 26:13431355, 1995.

RAIJ, B.van. New diagnostic techniques, universal soil extractants. Comm. Soil Sci. Plant Anal., 25:799-816, 1994.

RAIJ, B.van \& KUPPER, A. Capacidade de troca de cátions em solos: Estudo comparativo de alguns métodos. Bragantia, 25:327-336, 1966.

SHOEMAKER, H.E.; MACLEAN, E.O. \& PRATT, P.F. Buffer methods for determining lime requirement of soils with appreciable amounts of extractable aluminum. Soil Sci. Soc. Am. Proc., 25:274-277, 1961.

SKINNER, M.F.; ZABOWSKI, D.; HARRISON, R.; LOWE A. \& XUE, D. Measuring the cation exchange capacity of forest soils. Comm. Soil Sci. Plant Anal., 32:1751-1764, 2001.

SOUZA, I.G.; COSTA, A.C.S.; SAMBATTI, J.A.; PETERNELE, W.S.; TORMENA, C.A.; MONTES, C.R. \& CLEMENTE, C.A. Contribuição dos constituintes da fração argila de solos subtropicais à área superficial específica e à capacidade de troca catiônica. R. Bras. Ci. Solo, 31:1355$1365,2007$.

TEDESCO, M.J.; GIANELLO, C.; BISSANI, C.A.; BOHNEN, H. \& VOLKWEISS, S.J. Análises de solo, plantas e outros materiais. 2.ed. Porto Alegre, Universidade Federal do Rio Grande do Sul, 1995. 174p. (Boletim Técnico, 5)

TESSIER, A.; CAMPBELL, P. \& BISSON, M. Sequential extraction procedure for the speciation of particulate trace metals. Anal. Chem., 51:844-851, 1979.

VALLADARES, G.S.; PEREIRA, M.G.; SOUZA, J.M.P.F.; PÉREZ, D.V. \& ANJOS, L.H.C. Comparação entre os teores de nutrientes extraídos por três métodos em amostras de solos do Rio de Janeiro. R. Bras. Agroci., 7:137-141, 2001.

VILLIERS, J.M. \& JACKSON, M.L. Cation exchange capacity variations with $\mathrm{pH}$ in soil clays. Soil Sci. Soc. Am. Proc., 31:473-476, 1967. 
